\title{
Research Article \\ Potential Symmetry Studies on a Rotating Fluid System
}

\author{
S. Y. Lou, ${ }^{1,2,3}$ Man Jia, $^{1}$ F. Huang, ${ }^{4}$ and X. Y. Tang ${ }^{1,2}$ \\ ${ }^{1}$ Department of Physics, Ningbo University, Ningbo 315211, China \\ ${ }^{2}$ Department of Physics, Shanghai Jiao Tong University, Shanghai 200240, China \\ ${ }^{3}$ School of Mathematics, Fudan University, Shanghai 200433, China \\ ${ }^{4}$ Physical Oceanography Laboratory, Ocean University of China, Qingdao 266003, China
}

Correspondence should be addressed to S. Y. Lou, sylou@sjtu.edu.cn

Received 13 July 2011; Revised 26 September 2011; Accepted 26 October 2011

Academic Editor: Marc de Montigny

Copyright (C) 2012 S. Y. Lou et al. This is an open access article distributed under the Creative Commons Attribution License, which permits unrestricted use, distribution, and reproduction in any medium, provided the original work is properly cited.

A rotational fluid model which can be used to describe broad vortical flows ranging from large scale to the atmospheric mesoscale and the oceanic submesoscale is studied by the symmetry group theory. After introducing one scalar-, two vector-, and two tensor potentials, we find that the Lie symmetries of the extended system include many arbitrary functions of $z$ and $\{z, t\}$. The obtained Lie symmetries are used to find some types of exact solutions. One of exact solutions can be used to qualitatively describe the three-dimensional structure of hurricanes.

\section{Introduction}

Symmetry study is one of the effective methods to study complicated nonlinear problems. Though the Lie symmetry group method have been quite perfectly studied and some excellent books has been published [1], there still exist many important problems to be studied. For instance, to find group invariant solutions related to the generalized symmetries and nonlocal symmetries is still a very difficult topic. We know that for integrable systems there are infinitely many generalized symmetries [1-6] and nonlocal symmetries. The finite transformation (symmetry group) of the Lie symmetries may be obtained via Lie's first theorem. For many types of the nonlocal symmetries, the finite transformations can also be obtained by using Lie's first theorem; say, the Darboux transformations (DT) are just the finite transformation form of the nonlocal symmetries obtained from DT. This fact implies that some types of generalized symmetries and nonlocal symmetries can be localized to closed Lie symmetry algebra such that the Lie's first principle can be successfully applied. In this paper, we study a special type of nonlocal symmetries, potential symmetries of a 
rotating fluid model. The potential symmetries have been studied by many authors [79].

It is known that the quasi-geostrophic equations for a Boussinesq fluid in a uniformly rotating and stably stratified environment, which is an idealization for various phenomena in either atmosphere or ocean. In this paper, we consider the following rotating stratified fluid model $[10,11]$ :

$$
\begin{gathered}
u_{x}+v_{y}=0 \\
u_{t}+u u_{x}+v u_{y}-f v+p_{x}=0 \\
v_{t}+u v_{x}+v v_{y}+f u+p_{y}=0 \\
\rho+p_{z}=0 \\
\rho_{t}+u \rho_{x}+v \rho_{y}+w \rho_{z}=0
\end{gathered}
$$

where $f$ is the Coriolis parameter, $p$ is the pressure perturbation divided by a mean density $\rho_{0}, \rho$ is the density perturbation scaled by $\rho_{0} / g, u$ and $v$ are horizontal velocities, and $w$ is the vertical velocity.

It should be mentioned that the consistent condition of (1.2) and (1.3), $p_{x y}=p_{y x}$, is just the Euler equation for an ideal incompressible two-dimensional fluid. Some authors have studied the symmetry structure of the two-dimensional Euler equations [12-14].

In section 2, the Lie symmetries of the original model (1.1)-(1.5) are directly written down because it can be obtained by means of some known methods. Section 3 is devoted to discuss special types of generalized symmetries of the system (1.1)-(1.5) which are only Lie symmetries for the subsystem (1.1)-(1.3). In section 4, to find more symmetries and symmetry groups we discuss the potential symmetries which are Lie symmetries for an enlarged system. In section 5, the full symmetry group related to the Lie symmetries of the enlarged system is directly written down. The section 6 is devoted to find some new exact solutions which display abundant vortex structure and some of them qualitatively display some three-dimensional structure of hurricanes. The final section is a short summary and discussion.

\section{The Lie Symmetries of the System (1.1)-(1.5)}

A symmetry of (1.1)-(1.5),

$$
\sigma \equiv\left(\begin{array}{c}
\sigma^{u} \\
\sigma^{v} \\
\sigma^{p} \\
\sigma^{\rho} \\
\sigma^{w}
\end{array}\right),
$$


is defined as a solution of the linearized equations of (1.1)-(1.5):

$$
\begin{gathered}
\sigma_{x}^{u}+\sigma_{y}^{v}=0, \\
\sigma_{t}^{u}+\sigma^{u} u_{x}+u \sigma^{u} u_{x}+\sigma^{v} u_{y}+v \sigma^{u} u_{y}-f \sigma^{v}+\sigma_{x}^{p}=0, \\
\sigma_{t}^{v}+\sigma^{u} v_{x}+u \sigma_{x}^{v}+\sigma^{v} v_{y}+v \sigma_{y}^{v}+f \sigma^{u}+\sigma_{y}^{p}=0, \\
\sigma^{\rho}+\sigma_{z}^{p}=0 \\
\sigma_{t}^{\rho}+\sigma^{u} \rho_{x}+u \sigma_{x}^{\rho}+\sigma^{v} \rho_{y}+v \sigma_{y}^{\rho}+\sigma^{w} \rho_{z}+w \sigma_{z}^{\rho}=0,
\end{gathered}
$$

which means the model system is form invariant under the transformation

$$
\left(\begin{array}{c}
u \\
v \\
p \\
\rho \\
w
\end{array}\right) \rightarrow\left(\begin{array}{c}
u \\
v \\
p \\
\rho \\
w
\end{array}\right)+\epsilon\left(\begin{array}{c}
\sigma^{u} \\
\sigma^{v} \\
\sigma^{p} \\
\sigma^{\rho} \\
\sigma^{w}
\end{array}\right)
$$

with infinitesimal $\epsilon$. The Lie symmetries of (1.1)-(1.5) have the form

$$
\left(\begin{array}{c}
\sigma^{u} \\
\sigma^{v} \\
\sigma^{p} \\
\sigma^{\rho} \\
\sigma^{w}
\end{array}\right)=\left(\begin{array}{c}
U \\
V \\
P \\
Q \\
W
\end{array}\right)-X\left(\begin{array}{c}
u_{x} \\
v_{x} \\
p_{x} \\
\rho_{x} \\
w_{x}
\end{array}\right)-Y\left(\begin{array}{c}
u_{y} \\
v_{y} \\
p_{y} \\
\rho_{y} \\
w_{y}
\end{array}\right)-Z\left(\begin{array}{c}
u_{z} \\
v_{z} \\
p_{z} \\
\rho_{z} \\
w_{z}
\end{array}\right)-T\left(\begin{array}{c}
u_{t} \\
v_{t} \\
p_{t} \\
\rho_{t} \\
w_{t}
\end{array}\right),
$$

where $X, Y, Z, T, U, V, P, Q$, and $W$ are functions of $x, y, z, t, u, v, p, \rho$, and $w$.

Substituting (2.8) into (2.2)-(2.6), eliminating $u_{t}, v_{y}, p_{y}, \rho$, and $w$ via (1.1)-(1.5) and vanishing different powers of the fields $u, v, p, \rho, w$ and their derivatives, one can obtain the determining equations of the functions $X, Y, Z, T, U, V, P, Q$, and $W$. After solving these 
determining equations, one can find that the general Lie symmetries of (1.1)-(1.5) have the form (2.8) with

$$
\begin{gathered}
X=a_{1} x+\frac{1}{2}\left(a_{2} f t+2 a_{5}\right) y-a_{7}(t), \\
Y=a_{1} y-\frac{1}{2}\left(a_{2} f t+2 a_{5}\right) x-a_{8}(t), \\
Z=2\left(a_{1}-a_{2}\right) z+a_{4}, \\
T=a_{2} t+a_{3}, \\
U=-a_{7 t}+\left(a_{1}+a_{2}\right) u-\frac{1}{2}\left(-a_{2} f t-2 a_{5}\right) v+\frac{1}{2} a_{2} f y, \\
V=-a_{8 t}+\frac{1}{2}\left(-a_{2} f t-2 a_{5}\right) u+\left(a_{1}-a_{2}\right) v-\frac{1}{2} a_{2} f x, \\
P=\left(a_{7 t}-f a_{8}\right)_{t} x+\left(a_{7} f+a_{8 t}\right) y+2\left(a_{1}-a_{2}\right) p-\frac{1}{4} f^{2} a_{2}\left(y^{2}+x^{2}\right)+c_{1} z+a_{10}(t), \\
Q=-c_{1}, \\
W=\left(-3 a_{2}+2 a_{1}\right) w,
\end{gathered}
$$

which is a linear combination of the following generators:

(a) time translation ( $a_{3}$-part of (2.8) with (2.9))

$$
V_{1}=\partial_{t}
$$

(b) vertical space translation ( $a_{4}$-part of (2.8) with (2.9)),

$$
V_{2}=\partial_{z}
$$

(c) generalized $x$-translation and Galilean invariance

$$
V_{3}=-a_{7}(t) \partial_{x}-a_{7 t} \partial_{u}+\left(f a_{7 t} y+x a_{7 t t}\right) \partial_{p}
$$

which is $x$-translation for $a_{7}$ being constant and Galilean boost in $x$ direction for $a_{7} \sim t$

(d) generalized $y$-translation and Galilean invariance

$$
V_{4}=-a_{8}(t) \partial_{y}-a_{8 t} \partial_{v}+\left(f a_{8 t} x-y a_{8 t t}\right) \partial_{p},
$$


International Journal of Mathematics and Mathematical Sciences

which is $y$-translation for $a_{8}$ being constant and Galilean boost in $y$ direction for $a_{8} \sim t$;

(e) time and $z$-dependent $p$-translation (pressure shift for reference point)

$$
V_{5}=\left[c_{1} z+a_{10}(t)\right] \partial_{p}
$$

(f) space scaling invariance ( $a_{1}$-part of (2.8) with (2.9))

$$
V_{6}=x \partial_{x}+y \partial_{y}+2 z \partial_{z}+u \partial_{u}+v \partial_{v}+2 p \partial_{p}+2 w \partial_{w}
$$

(g) time-independent rotation ( $a_{5}$-part of (2.8) with (2.9))

$$
V_{7}=-y \partial_{x}+x \partial_{y}-v \partial_{u}+u \partial_{v}
$$

(h) time scaling company with time-dependent rotation ( $a_{2}$-part of (2.8) with (2.9))

$$
\begin{aligned}
V_{8}= & \frac{1}{2} t f y \partial_{x}-\frac{1}{2} t f x \partial_{y}+t \partial_{t}-2 z \partial_{z}+\left(\frac{f}{2}(y+v t)-u\right) v \partial_{u} \\
& -\left(v+\frac{f}{2}(x+u t)\right) \partial_{v}-\left(2 p+\frac{f^{2}}{4}\left(x^{2}+y^{2}\right)\right) \partial_{p}-3 w \partial_{w}
\end{aligned}
$$

where $a_{i}, i=1, \ldots, 5$, and $c_{1}$ are arbitrary constants while $a_{7}, a_{8}$, and $a_{9}$ are arbitrary functions of $t$.

It should be pointed out that the time scaling (2.17) is linked with the time-dependent rotation. This property may hint us that we should uncover more symmetries of the model.

\section{The Lie Symmetries of the Subsystem (1.1)-(1.3)}

From (1.1)-(1.3), we know that the fields $u, v$, and $p$ constitute a closed subsystem. Whence $u, v$, and $p$ are fixed, the density $\rho$ and the vertical velocity $w$ can be simply obtained from (1.4) and (1.5), respectively, via differentiations.

Therefore, in this section we study the Lie symmetries of the subsystem (1.1)-(1.3) which is a solution of (2.2)-(2.4) with the solution form

$$
\left(\begin{array}{c}
\sigma^{u} \\
\sigma^{v} \\
\sigma^{p}
\end{array}\right)=X\left(\begin{array}{c}
u_{x} \\
v_{x} \\
p_{x}
\end{array}\right)+Y\left(\begin{array}{c}
u_{y} \\
v_{y} \\
p_{y}
\end{array}\right)+Z\left(\begin{array}{c}
u_{z} \\
v_{z} \\
p_{z}
\end{array}\right)+T\left(\begin{array}{c}
u_{t} \\
v_{t} \\
p_{t}
\end{array}\right)-\left(\begin{array}{c}
U \\
V \\
P
\end{array}\right)
$$


where $X, Y, Z, T, U, V$, and $P$ are functions of $x, y, z, t, u, v$, and $p$. The same procedure as the last section leads to the general solution

$$
\begin{gathered}
X=x a_{1}+\frac{1}{2} a_{2} y f t+y a_{5}-a_{7}, \\
Y=-\frac{1}{2} a_{2} x f t-x a_{5}+y a_{1}-a_{8}, \\
Z=a_{4}(z), \\
T=a_{2} t+a_{3}, \\
U=\left(a_{1}-a_{2}\right) u+\frac{1}{2}\left(t f a_{2}+2 a_{5}\right) v+\frac{1}{2} a_{2} f y-a_{7 t}, \\
V=\frac{1}{2}\left(-2 a_{5}-t f a_{2}\right) u+\left(a_{1}-a_{2}\right) v-\frac{1}{2} a_{2} f x-a_{8 t}, \\
P=2\left(a_{1}-a_{2}\right) p-\frac{1}{4} a_{2}\left(y^{2}+x^{2}\right) f^{2}-\left(x a_{7 t}-y a_{8 t}\right) f+x a_{7 t t}-y a_{8 t t}+a_{9},
\end{gathered}
$$

where $a_{i}, i=1, \ldots, 5$, are arbitrary functions of $z$ and $a_{7}, a_{8}$ and $a_{9}$ are arbitrary functions of $\{z, t\}$. It is clear that the Lie symmetries of the last section are just the special case of (3.2) for $a_{1}, a_{2}, a_{3}, a_{5}$ being arbitrary constants, $a_{4}$ and $a_{9}$ being linear functions of $z$, and $a_{7}$ and $a_{8}$ being only functions of $t$.

Because of the entrance of arbitrary functions, the Lie symmetries (3.1) of the subsystem (1.1)-(1.3) become the generalized symmetries for the full system (1.1)-(1.5). The generalized symmetries of (1.1)-(1.5) are expressed by (2.8) with (3.2) and

$$
\begin{aligned}
Q= & \left(a_{2} t+a_{3}\right)_{z} p_{t}+\left(x a_{1}+\frac{1}{2} a_{2} y f t+y a_{5}-a_{7}\right)_{z} p_{x}+\left(y a_{1}-\frac{1}{2} a_{2} x f t-x a_{5}-a_{8}\right)_{z} p_{y} \\
+ & \left(-x a_{7 t t}-y a_{8 t t}-y f a_{7 t}+x f a_{8 t}-a_{10}+\frac{1}{4} a_{2}\left(y^{2}+x^{2}\right) f^{2}\right)_{z} \\
& -a_{4 z} \rho-2\left(a_{1}-a_{2}\right)_{z} p+2\left(a_{1}-a_{2}\right) \rho, \\
W= & \left\{2 a_{4 z}-2 a_{1}+2 a_{2}-p_{z z}^{-1}\right. \\
\quad & {\left[-2 T_{z} p_{z t}-2 X_{z} p_{x z}-2 Y_{z} p_{y z}-X_{z z} p_{x}-Y_{z z} p_{y}-T_{z z} p_{t}\right.} \\
& +\left(4 a_{2 z}+a_{4 z z}-4 a_{1 z}\right) \rho+2\left(a_{1 z z}-a_{2 z z}\right) p-\frac{1}{4}\left(y^{2}+x^{2}\right) f^{2} a_{2 z z}+y f a_{7 z z t}-x f a_{8 z z t} \\
& \left.\left.+x a_{7 z z t t}+y a_{8 z z t t}+p_{0 z z}\right]\right\} w
\end{aligned}
$$


International Journal of Mathematics and Mathematical Sciences

$$
\begin{aligned}
& -\left\{v f a_{7 z t}-u f a_{8 z t}-p_{y y}\left(v y a_{1 z}-v x a_{5 z}-v a_{8 z}\right)\right. \\
& +x a_{7 z t t t}+y a_{8 z t t t}+\left[v\left(2 a_{1}-3 a_{2}\right)-v a_{4 z}\right] p_{y z}+\left(2 a_{1}-a_{4 z}-3 a_{2}\right) p_{z t}+(y f+u) a_{7 z t t} \\
& -(x f-v) a_{8 z t t}+\left(2 a_{1}-3 a_{2}\right)_{z} p_{t}-\left(a_{2} t+a_{3}\right)_{z} p_{t t}+p_{0 z t}-\frac{1}{2}\left(u x f+v y f-p_{y y} v x t\right) f a_{2 z} \\
& +\left[-y a_{5 z}+a_{7 z}-u a_{4 z}-x a_{1 z}-\frac{1}{2} y f a_{2 z} t-u a_{3 z}-u a_{2 z} t+u\left(2 a_{1}-3 a_{2}\right)\right] p_{x z} \\
& -\left[(y v-u x) a_{5 z}+v a_{7 z}+u a_{8 z}-(v x+u y) a_{1 z}+\frac{1}{2} t(u x-y v) f a_{2 z}\right] p_{x y} \\
& +\left[u a_{1 z}-\frac{1}{2}(v t+y) f a_{2 z}-v a_{5 z}+a_{7 z t}-2 u a_{2 z}\right] p_{x} \\
& +\left[a_{1 z} v+\frac{1}{2}(u t+x) f a_{2 z}+u a_{5 z}-2 v a_{2 z}+a_{8 z t}\right] p_{y} \\
& +u\left(-y a_{5 z}-x a_{1 z}-\frac{1}{2} y f a_{2 z} t+a_{7 z}\right) p_{x x} \\
& \left.+\left(\frac{1}{2} x f a_{2 z} t+a_{8 z}+x a_{5 z}-y a_{1 z}-v a_{3 z}-v a_{2 z} t\right) p_{y t}\right\} p_{z z}^{-1} .
\end{aligned}
$$

In addition to the symmetries (2.8) with (3.3) are not point symmetries, the time scaling invariance related to $a_{2}=a_{2}(z)$

$$
\begin{gathered}
V=a_{2} t \partial_{t}+\frac{1}{2} a_{2} y f t \partial_{x}-\frac{1}{2} a_{2} x f t \partial_{y}+\frac{1}{2} a_{2}(t f v-2 u+f y) \partial_{u}-\frac{1}{2} a_{2}(t f u+2 v+f x) \partial_{v} \\
-\frac{1}{4} a_{2}\left[8 p+\left(y^{2}+x^{2}\right) f^{2}\right] \partial_{p}+\frac{1}{4} a_{2 z}\left[4 t p_{t}+2 y f t p_{x}-2 x f t p_{y}+\left(y^{2}+x^{2}\right) f^{2}\right] \partial_{\rho} \\
+\left\{\left[2 a_{2}-p_{z z}^{-1}\left[a_{2 z}\left(x f t p_{y z}-2 t p_{z t}-y f t p_{x z}+4 \rho\right)+\frac{1}{2} a_{2 z z}\left(x f t p_{y}-t y f p_{x}-2 t p_{t}\right)\right.\right.\right. \\
\left.\left.-a_{2 z z}\left(2 p+\frac{1}{4}\left(y^{2}+x^{2}\right) f^{2}\right)\right]\right] w \\
-\left[\frac{1}{2}\left(p_{y y} v x t-u x f-v y f\right) f a_{2 z}-3 a_{2}\left(p_{y z}+p_{z t}\right)-a_{2 z}\left(3 p_{t}+t p_{t t}\right)\right. \\
-\frac{1}{2}\left[(y f+2 u) a_{2 z} t+6 a_{2} u\right] p_{x z}+\frac{1}{2} t(u x-y v) f a_{2 z} p_{x y}-\frac{1}{2}[(v t+y) f+4 u] a_{2 z} p_{x} \\
\left.\left.+\frac{1}{2}[(u t+x) f-4 v] a_{2 z} p_{y}-\frac{1}{2} u y f a_{2 z} t p_{x x}+\frac{1}{2} a_{2 z}(x f t-2 v t) p_{y t}\right] p_{z z}^{-1}\right\} \partial_{w}
\end{gathered}
$$

is still companied by the time-dependent rotation. 


\section{Potential Symmetries of (1.1)-(1.5)}

From (1.1), we know that it is natural to introduce a special scalar potential, the stream function $\psi$, such that

$$
u=-\psi_{y}, \quad v=\psi_{x} .
$$

We call a symmetry potential symmetry if it is explicitly dependent on a potential, say, $\psi$, (or its derivatives) for the fields $u, v, p, \rho$, and $w$ described by (1.1)-(1.5). Obviously, a potential symmetry for the field system $\{u, v, p, \rho, w\}$ is a nonlocal one though it may be a Lie symmetry for the enlarged system $\{u, v, p, \rho, w, \psi\}$. From the general symmetry expression (3.3), we know that it is useful to introduce the following potential vector fields

$$
\begin{gathered}
\vec{v}=\left(\psi_{x}, \psi_{y}, \psi_{z}, \psi_{t}\right)=\left(v_{1}=v, v_{2}=-u, v_{3}, v_{4}\right), \\
\vec{f}=\left(p_{x}, p_{y}, p_{z}, p_{t}\right)=\left(f_{1}, f_{2}, f_{3}=-\rho, f_{4}\right),
\end{gathered}
$$

and symmetric potential tensor fields

$$
\begin{gathered}
\psi_{i j}=\psi_{j i}=\psi_{x_{i} x_{j}}, \quad i, j=1,2,3,4, x_{1}=x, x_{2}=y, x_{3}=z, x_{4}=t \\
p_{i j}=p_{j i}=p_{x_{i} x_{j}}, \quad i, j=1,2,3,4 .
\end{gathered}
$$

Now using the standard method as the last two sections, we can obtain the Lie symmetries of the enlarged system (1.1)-(1.5) and (4.1)-(39) for thirty-one fields $u=-v_{2}, v=$ $v_{1}, p, w, \psi, v_{3}, v_{4}, f_{1}, f_{2}, \rho=-f_{3}, f_{4}, \psi_{i j}$ and $p_{i j}, i \leq j=1,2,3,4$. The result has the following generators $\left\{V_{1}, V_{2}, V_{3}, V_{4}, V_{5}, V_{6}, V_{7}, V_{8}, V_{9}, V_{10}\right\} \equiv \mho$ for the original fields $u, v, w, p, \rho$ and the potential $\psi$ :

$$
\begin{aligned}
& V_{1}=a_{1}\left(x \partial_{x}+y \partial_{y}+2 \psi \partial_{\psi}+u \partial_{u}+v \partial_{v}+2 p \partial_{p}+2 \rho \partial_{\rho}\right)+a_{1 z} \\
& \times\left\{\left(x f_{1}+y f_{2}-2 p\right) \partial_{\rho}+p_{33}^{-1}\right. \\
& \times\left[2 w\left(2 \rho+x p_{13}+y p_{23}\right)+u\left(x p_{11}+y p_{12}-f_{1}\right)\right. \\
& \left.\left.+v\left(x p_{12}+y p_{22}-f_{2}\right)-2 f_{4}+x P_{13}+y p_{24}\right] \partial_{w}\right\}+a_{1 z z} p_{33}^{-1} w\left(x f_{1}+y f_{2}-2 p\right) \partial_{w} \\
& V_{2}=a_{2}\left[t \partial_{t}-\psi \partial_{\psi}-u \partial_{u}-v \partial_{v}+(f \psi-2 p) \partial_{p}-\left(f v_{3}+2 \rho\right) \partial_{\rho}\right. \\
& \left.+\left(w+f p_{33}^{-1}\left(w \psi_{33}+\psi_{34}+u \psi_{13}+v \psi_{23}\right)\right) \partial_{w}\right]+a_{2 z}\left(t f_{4}-f \psi+2 p\right) \partial_{\rho} \\
& +a_{2 z}\left\{2 t w^{2}+p_{33}^{-1}\left[2 w\left(2 \rho+f v_{3}+t u p_{13}+t v p_{23}\right)-u\left(2 f_{1}+t p_{14}\right)\right.\right. \\
& \left.\left.-v\left(2 f_{2}+t p_{24}\right)+v_{4} f-3 f_{4}-t p_{44}\right]\right\} \partial_{w}+a_{2 z z} p_{33}^{-1} w\left(f \psi-2 p-t f_{4}\right) \partial_{w},
\end{aligned}
$$




$$
\begin{aligned}
& V_{3}=a_{3} \partial_{t}+a_{3 z}\left\{f_{4} \partial_{\rho}+\left[2 w^{2}+p_{33}^{-1}\left(2 w\left(u p_{13}+v p_{23}\right)-u p_{14}-v p_{24}-p_{44}\right)\right] \partial_{w}\right\} \\
& -a_{3 z z} p_{33}^{-1} w f_{4} \partial_{w} \\
& V_{4}=a_{4} \partial_{z}-a_{4 z}\left(\rho \partial_{\rho}+w \partial_{w}\right)+a_{4 z z} p_{33}^{-1} w \rho \partial_{w}, \\
& V_{5}=-a_{5}\left[x \partial_{y}-y \partial_{x}-v \partial_{u}+u \partial_{v}\right] \\
& +a_{5 z}\left\{\left(x f_{2}-y f_{1}\right) \partial_{\rho}+p_{33}^{-1}\left[2\left(y p_{13}-x p_{23}\right) w\right.\right. \\
& \left.\left.+\left(y p_{11}-x p_{12}-f_{2}\right) u+\left(f_{1}+y p_{12}-x p_{22}\right) v+y p_{14}-x p_{24}\right] \partial_{w}\right\} \\
& +a_{5 z z} p_{33}^{-1}\left(y f_{1}-x f_{2}\right) w \partial_{w} \\
& V_{6}=-a_{6}\left[x t \partial_{y}-y t \partial_{x}+\frac{1}{2}\left(x^{2}+y^{2}\right) \partial_{\psi}-(v t+y) \partial_{u}+(u t+x) \partial_{v}+\frac{1}{2}\left(4 \psi+f x^{2}+f y^{2}\right) \partial_{p}\right. \\
& \left.-2 v_{3} \partial_{\rho}+\frac{2}{p_{33}}\left(u \psi_{13}+v \psi_{23}+w \psi_{33}+\psi_{34}\right) \partial_{w}\right] \\
& -a_{6 z}\left\{\frac{1}{2}\left(2 t x f_{2}-2 t y x_{1}-4 \psi-f x^{2}-f y^{2}\right) \partial_{\rho}\right. \\
& +p_{33}^{-1}\left[u\left(f x-f_{2} t-x t p_{12}+y t p_{11}\right)+v\left(y f+t f_{1}-x t p_{22}+y t p_{12}\right)\right. \\
& \left.\left.+2 w\left(2 v_{3}+y t p_{13}-x t p_{23}\right)+y f_{1}+2 v_{4}-x t p_{24}+y t p_{14}-x f_{2}\right] \partial_{w}\right\} \\
& -\frac{1}{2} a_{6 z z} p_{33}^{-1}\left(4 \psi+2 y t f_{1}-2 x t f_{2}+f x^{2}+f y^{2}\right) w \partial_{w} \\
& V_{7}=-a_{7} \partial_{x}-a_{7 t}\left(-y \partial_{\psi}+\partial_{u}-f y \partial_{p}\right)-a_{7 t t} x \partial_{p} \\
& -a_{7 z}\left(f_{1} \partial_{\rho}-p_{33}^{-1}\left(2 w p_{13}+u p_{11}+p_{14}+v p_{12}\right) \partial_{w}\right) \\
& +a_{7 z t}\left[f y \partial_{\rho}-p_{33}^{-1}\left(f_{1}+f v\right) \partial_{w}\right]+a_{7 z t t} p_{33}^{-1}(u+f y) \partial_{w} \\
& -a_{7 z z t}\left(x \partial_{\rho}-p_{33}^{-1} w y f \partial_{w}\right)+\left(a_{7 z t t t} x+a_{7 z z t t} x w+a_{7 z z} f_{1} w\right) p_{33}^{-1} \partial_{w} \\
& V_{8}=-a_{8} \partial_{y}-a_{8 t}\left(x \partial_{\psi}+\partial_{v}+x f \partial_{p}\right)+\left(a_{8 z z} w f_{2}+a_{8 z t t t} y-a_{8 z z t} w x f\right) p_{33}^{-1} \partial_{w} \\
& +a_{8 t t} y \partial_{p}-a_{8 z}\left[f_{2} \partial_{\rho}-p_{33}^{-1}\left(2 w p_{23}+v p_{22}+p_{24}+u p_{12}\right) \partial_{w}\right]+a_{8 z z t t} p_{33}^{-1} y w \partial_{w} \\
& +a_{8 z t}\left[f x \partial_{\rho}+p_{33}^{-1}\left(f_{2}-f u\right) \partial_{w}\right]-a_{8 z t t}\left[y \partial_{\rho}-p_{33}^{-1}(v-x f) \partial_{w}\right], \\
& V_{9}=a_{9} \partial_{p}-a_{9 z} \partial_{\rho}-\left(a_{9 t t} w+a_{9 z t}\right) p_{33}^{-1} \partial_{w} \\
& V_{10}=a_{10} \partial_{\psi}
\end{aligned}
$$


where $a_{i}, i=1, \ldots, 6$, are arbitrary functions of $z$ and $a_{7}, a_{8}, a_{9}, a_{10}$ are arbitrary functions of $\{z, t\}$.

Remark 4.1. $z$-dependent time scaling symmetry $V_{2}$ given by (4.5) is independent of the timedependent rotation symmetry (4.9) thanks to the inclusion of the potential symmetry.

Remark 4.2. The symmetries $V_{2}$ and $V_{6}$ are nonlocal symmetries of the original system $\{u, v, p, \rho, w\}$ while they are local Lie symmetries for the enlarged system.

Remark 4.3. All the symmetries except $V_{10}$ are generalized symmetries for the original system and they all become the Lie symmetries for the enlarged system.

\section{Finite Transformation Group of the System (1.1)-(1.5) Related to Lie Symmetries of the Enlarged System}

To find the finite transformation group of the system (1.1)-(1.5) related to the Lie symmetry algebra $\mho$, we have to solve the initial valued problem

$$
\begin{gathered}
\frac{\mathrm{d} x^{\prime}}{X^{\prime}}=\frac{\mathrm{d} y^{\prime}}{Y^{\prime}}=\frac{\mathrm{d} z^{\prime}}{Z^{\prime}}=\frac{\mathrm{d} t^{\prime}}{T^{\prime}}=\frac{\mathrm{d} \psi^{\prime}}{\Psi^{\prime}}=\frac{\mathrm{d} u^{\prime}}{U^{\prime}}+\frac{\mathrm{d} v^{\prime}}{V^{\prime}}=\frac{\mathrm{d} w^{\prime}}{W^{\prime}}=\frac{\mathrm{d} p^{\prime}}{P^{\prime}}=\cdots=d \epsilon, \\
\left.\left\{x^{\prime}, y^{\prime}, z^{\prime}, t^{\prime}, \psi^{\prime}, u^{\prime}, v^{\prime}, w^{\prime}, p^{\prime}, \ldots\right\}\right|_{e=0}=\{x, y, z, t, \psi, u, v, w, p, \ldots\},
\end{gathered}
$$

where $x^{\prime}, y^{\prime}, z^{\prime}, t^{\prime}, u^{\prime}, v^{\prime}, w^{\prime}, p^{\prime}, \ldots$, are functions of $\epsilon$ and $X^{\prime}, Y^{\prime}, Z^{\prime}, T^{\prime}, U^{\prime}, V^{\prime}, P^{\prime}, \ldots$ can be read off from the linear combination of the generators of the algebra $\mho$. For instance,

$$
\begin{gathered}
X^{\prime}=-a_{7}^{\prime}+a_{1}^{\prime} x^{\prime}-t_{2}^{\prime} y^{\prime}-g^{\prime} y^{\prime} t^{\prime}, \\
Y^{\prime}=-a_{8}^{\prime}+a_{1}^{\prime} y^{\prime}+t_{2}^{\prime} x^{\prime}+g^{\prime} x^{\prime} t^{\prime}, \\
Z^{\prime}=a_{4}^{\prime}\left(z^{\prime}\right), \\
T^{\prime}=t_{0}^{\prime}+t^{\prime} t_{1}^{\prime}, \\
U^{\prime}=-a_{7 t^{\prime}}^{\prime}+\left(c_{2}^{\prime}-t_{1}^{\prime}\right) u^{\prime}-t_{2}^{\prime} v^{\prime}-g^{\prime}\left(v^{\prime} t^{\prime}+y^{\prime}\right), \\
V^{\prime}=-a_{8 t^{\prime}}^{\prime}+\left(c_{2}^{\prime}-t_{1}^{\prime}\right) v^{\prime}+t_{2}^{\prime} u^{\prime}+g^{\prime}\left(u^{\prime} t^{\prime}+x^{\prime}\right), \\
P^{\prime}=f a_{7 t^{\prime}}^{\prime} y^{\prime}-x^{\prime} a_{7 t^{\prime} t^{\prime}}^{\prime}-f a_{8 t^{\prime}}^{\prime} x^{\prime}+y^{\prime} a_{8 t^{\prime} t^{\prime},}^{\prime}
\end{gathered}
$$

with $\left\{a_{1}^{\prime} \equiv a_{1}\left(z^{\prime}\right), a_{3}^{\prime} \equiv a_{3}\left(z^{\prime}\right), a_{2}^{\prime} \equiv a_{2}\left(z^{\prime}\right), a_{5}^{\prime} \equiv a_{5}\left(z^{\prime}\right), g^{\prime} \equiv g\left(z^{\prime}\right), a_{4}^{\prime} \equiv a_{4}\left(z^{\prime}\right), \ldots\right\}$ being arbitrary functions of $z^{\prime}$ and $\left\{a_{7}^{\prime} \equiv a_{7}\left(z^{\prime}, t^{\prime}\right), a_{8}^{\prime} \equiv a_{8}\left(z^{\prime}, t^{\prime}\right), \ldots\right\}$ being arbitrary functions of the indicated variables.

Because the density $\rho$ and the vertical velocity $w$ can be simply obtained from (1.4) and (1.5) when the fields $\{u, v, p, \psi\}$ are known, we write down only the transformation group theorem on the fields $\{u, v, p, \psi\}$ here. 
Theorem 5.1. If $\{U(x, y, z, t), V(x, y, z, t), P(x, y, z, t), \Psi(x, y, z, t)\}$ is a solution of the system (1.1)-(1.3) and (4.1), so is $\{u, v, p, \psi\}$ with

$$
\begin{aligned}
u= & -\alpha_{6} y-\alpha_{8 t} \sin \theta+\alpha_{7 t} \cos \theta+\alpha_{2} \alpha_{1}^{-1}[U(\xi, \eta, \zeta, \tau) \cos \theta-V(\xi, \eta, \zeta, \tau) \sin \theta], \\
v= & \alpha_{6} x+\alpha_{7 t} \sin \theta+\alpha_{8 t} \cos \theta+\alpha_{2} \alpha_{1}^{-1}[V(\xi, \eta, \zeta, \tau) \cos \theta+U(\xi, \eta, \zeta, \tau) \sin \theta], \\
p= & \frac{\alpha_{2}}{\alpha_{1}^{2}}\left[\alpha_{2} P(\xi, \eta, \zeta, \tau)+\left(f+2 \alpha_{6}-f \alpha_{2}\right) \Psi(\xi, \eta, \zeta, \tau)\right]+\alpha_{9}+\frac{\alpha_{6}}{2}\left(f+\alpha_{6}\right)\left(x^{2}+y^{2}\right) \\
& +(x \cos \theta+y \sin \theta)\left[\left(f+2 \alpha_{6}\right) \alpha_{8 t}-\alpha_{7 t t}\right]+(x \sin \theta-y \cos \theta)\left[\left(f+2 \alpha_{6}\right) \alpha_{7 t}+\alpha_{8 t t}\right], \\
\psi= & \frac{\alpha_{2}}{\alpha_{1}^{2}} \Psi(\xi, \eta, \zeta, \tau)+\alpha_{10}+\frac{\alpha_{6}}{2}\left[\left(y \cos \left(\alpha_{6} t\right)-x \sin \left(\alpha_{6} t\right)\right)^{2}+\left(y \sin \left(\alpha_{6} t\right)+x \cos \left(\alpha_{6} t\right)\right)^{2}\right] \\
& +x\left[\alpha_{8 t} \cos \theta+\alpha_{7 t} \sin \theta\right]+y\left[\alpha_{8 t} \sin \theta-\alpha_{7 t} \cos \theta\right],
\end{aligned}
$$

where $\xi=\alpha_{1}\left(y \sin \theta+x \cos \theta-\alpha_{7}\right), \eta=\alpha_{1}\left(y \cos \theta-x \sin \theta-\alpha_{8}\right), \zeta=\alpha_{4}, \tau=\alpha_{2} t+\alpha_{3}, \theta \equiv$ $\alpha_{6} t+\alpha_{5},\left\{\alpha_{1}, \alpha_{2}, \alpha_{3}, \alpha_{4}, \alpha_{5}\right\}$ and $\alpha_{6}$ are arbitrary functions of $z$ while $\left\{\alpha_{7}, \alpha_{8}, \alpha_{9}\right\}$ and $\alpha_{10}$ are arbitrary functions of $\{z, t\}$.

To prove the theorem, one can solve the initial problem (5.1) or directly substitute (5.3) to (1.1)-(1.3) and (4.1). Actually, it is more straightforward to verify that the infinitesimal form of (5.3) is just the linear combination of the generators given in the last section by taking

$$
\alpha_{1} \longrightarrow 1+\epsilon a_{1}, \quad \alpha_{2} \longrightarrow 1+\epsilon a_{2}, \quad \alpha_{4} \longrightarrow z+\epsilon a_{4}, \quad \alpha_{i} \longrightarrow \epsilon a_{i}, \quad i \neq 1,2,4,
$$

with the infinitesimal parameter $\epsilon$.

\section{Exact Solutions and Applications}

To find some types of new exact solutions via the finite symmetry group theorem of the last section, we should find some simple exact seed solutions at first. Fortunately, it is not difficult to verify that the model (1.1)-(1.5) possesses the following known trivial solution:

$$
\begin{gathered}
u=-2 F(r, z) y, \quad r \equiv x^{2}+y^{2}, \\
v=2 x F(r, z), \\
p=\int F(r, z)(2 F(r, z)+f) d r, \\
\rho=-\int F_{z}(r, z)(4 F(r, z)+f) d r, \\
w=-\frac{1}{p_{z z}}\left(p_{z t}+u p_{x z}+v p_{y z}\right),
\end{gathered}
$$

with arbitrary function $F \equiv F(r, z)$ of $\{r, z\}$. 
Applying the group theorem of the last section to solution (6.1), we have

$$
\begin{aligned}
u= & -\alpha_{6} y-\alpha_{8 t} \sin \theta+\alpha_{7 t} \cos \theta-2 \alpha_{2} \alpha_{1}^{-1}\left[\eta F\left(R, \alpha_{4}\right) \cos \theta+\xi F\left(R, \alpha_{4}\right) \sin \theta\right] \\
v= & \alpha_{6} x+\alpha_{7 t} \sin \theta+\alpha_{8 t} \cos \theta+2 \alpha_{2} \alpha_{1}^{-1}\left[\xi F\left(R, \alpha_{4}\right) \cos \theta-\eta F\left(R, \alpha_{4}\right) \sin \theta\right] \\
p= & \frac{\alpha_{2}}{\alpha_{1}^{2}}\left[\alpha_{2} \int F\left(R, \alpha_{4}\right)\left(2 F\left(R, \alpha_{4}\right)+f\right) d R+\left(f+2 \alpha_{6}-f \alpha_{2}\right) \int F\left(R, \alpha_{4}\right) d R\right]+\alpha_{10} \\
& +\frac{\alpha_{6}}{2}\left(f+\alpha_{6}\right)\left(x^{2}+y^{2}\right)+(x \cos \theta+y \sin \theta)\left[\left(f+2 \alpha_{6}\right) \alpha_{8 t}-\alpha_{7 t t}\right] \\
& +(x \sin \theta-y \cos \theta)\left[\left(f+2 \alpha_{6}\right) \alpha_{7 t}+\alpha_{8 t t}\right] \\
\rho= & -p_{z \prime} \\
w= & -\frac{1}{p_{z z}}\left(p_{z t}+u p_{x z}+v p_{y z}\right)
\end{aligned}
$$

with

$$
R \equiv \alpha_{1}^{2}\left[\left(y \sin \theta+x \cos \theta-\alpha_{7}\right)^{2}+\left(y \cos \theta-x \sin \theta-\alpha_{8}\right)^{2}\right]
$$

The different selections of the arbitrary functions appeared in the exact solution (6.2) may lead to various vortex and circumfluence structures. It was demonstrated that the vortex and circumfluence solutions could be applied to tropical cyclones. A concrete application of a two-dimensional vortex solution to Hurricane Katrina 2005 was discussed in [16]. Similar to the two dimensional case, solution (6.2) displays some interesting phenomena. The first part, $u \sim-\alpha_{6} y, v \sim \alpha_{6} x$ and the $F\left(R, \alpha_{4}\right)$ parts of the solution exhibit abundant vortex structure. $\left\{x=\alpha_{7}, y=\alpha_{8}\right\}$ is expressed as the center of the vortices while the second part $\left(\left\{u \sim-\alpha_{8 t} \sin \theta+\alpha_{7 t} \cos \theta, v \sim \alpha_{7 t} \sin \theta+\alpha_{8 t} \cos \theta\right\}\right)$ of the solution shows us that the induced flow (the background wind) is related to the moving of the vortex center. This fact can be used to predict regular cyclone's track [16]. In order to find possible three-dimensional structure of the cyclones, one has to fix the arbitrary functions. Here we just take a special form of (6.2),

$$
\begin{gathered}
u=-F\left(R_{1}, z\right) y, \quad R_{1} \equiv x^{2}+y^{2}, \quad v=F\left(R_{1}, z\right) x, \\
w=-\frac{\alpha_{10 z t}(z, t)}{\int\left[\left(4 F\left(R_{1}, z\right)+f\right) F_{z z}\left(R_{1}, z\right)+4 F_{z}^{2}\left(R_{1}, z\right)^{2}\right] \mathrm{d} R_{1}+\alpha_{10 z z}(z, t)},
\end{gathered}
$$

with only two arbitrary functions $F\left(R_{1}, z\right)$ and $\alpha_{10}(z, t)$ to qualitatively display the threedimensional vortex structure. 
For the sake of capturing the known features of cyclone's structure, we here assume the arbitrary functions to be a more special form such that

$$
\begin{aligned}
& u=-c_{0} y \operatorname{sech}\left(c_{1} z+c_{5}\right) \tanh \left(c_{1} z+c_{5}\right) \operatorname{sech}\left(\frac{R_{1}}{c_{2}}\right), \\
& v=c_{0} x \operatorname{sech}\left(c_{1} z+c_{5}\right) \tanh \left(c_{1} z+c_{5}\right) \operatorname{sech}\left(\frac{R_{1}}{c_{2}}\right), \\
& w=c_{4}\left[2 c_{3}+c_{0}^{2} c_{1}^{2} c_{2} \operatorname{sech}^{2}\left(c_{1} z+c_{5}\right)\left(10 \operatorname{sech}^{4}\left(c_{1} z+c_{5}\right)-11 \operatorname{sech}^{2}\left(c_{1} z+c_{5}\right)+2\right) \tanh \left(\frac{R_{1}}{c_{2}}\right)\right. \\
& \left.\quad+c_{0} c_{1}^{2} c_{2} f \tanh \left(c_{1} z+c_{5}\right) \operatorname{sech}\left(c_{1} z+c_{5}\right)\left(1-6 \operatorname{sech}^{2}\left(c_{1} z+c_{5}\right)\right) \arctan \left(\exp \left(\frac{R_{1}}{c_{2}}\right)\right)\right]^{-1},
\end{aligned}
$$

with arbitrary constants $c_{i}, i=0,1,2, \ldots, 5$.

For simplicity, in the special selection, we have set the cyclone's center to be located at $(0,0, z)$. The parameters $c_{2}$ and $c_{1}$ determine typhoon's horizontal and vertical scales, respectively. The sign of $c_{0}$ decides the rotating direction of the vortex. The value of $-c_{5} / c_{1}$ gives the turning plane where typhoon changes its rotating direction, namely, from cyclone to anticyclone.

The velocity field of a cyclone is plotted in Figure 1 with (6.5)-(6.8) for the fixed parameters

$$
c_{0}=c_{5}=-1, \quad c_{1}=10, \quad c_{3}=1000, \quad c_{4}=f=1, \quad c_{2}=5 .
$$

Figure 1(a) shows the vortex structure rotating anticlockwise in lower level. While the rotation becomes clockwise at higher altitude, as observed in Figure 1(b). It is clear from Figure 1(c) that around the high $z=0.1$, the atmospheric flow turns from cyclone to anticyclone, which is one of the typical characters of a cyclone. It means that the energy moves inside at the lower layer and then radiates at the higher. Besides, the cyclone center and radius can be easily distinguished in the profiles of the velocity field.

The corresponding three-dimensional structure is depicted in Figure 2. In order to make it more clear how the atmosphere flows to form a cyclone, one can solve a simple system of equations coming from the relations

$$
\frac{\mathrm{d} x}{\mathrm{~d} t}=u, \quad \frac{\mathrm{d} y}{\mathrm{~d} t}=v, \quad \frac{\mathrm{d} z}{\mathrm{~d} t}=w .
$$

Since it is difficult to obtain an analytical solution of system (6.9), we solve it numerically with the initial conditions $x(0)=z(0)=0$ and $y(0)=2$. It is obviously revealed from the line depicted in Figure 2 that typhoon has a spiral pattern. The air close to the ground spirals upward and anticlockwise and then changes its rotation direction when approaching a higher altitude, which is consistent with the real observations. One can see a much closer comparison of our analytical results with a recent high-resolution hurricane simulation picture (Figure 3) using the WRF-ARW [15]. 


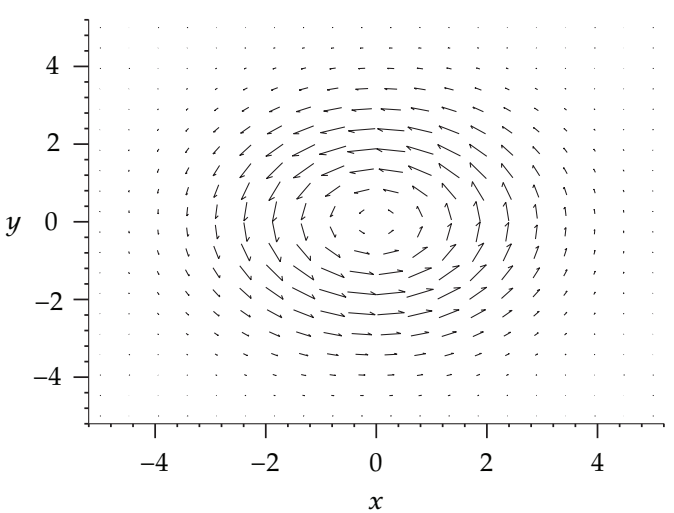

(a)

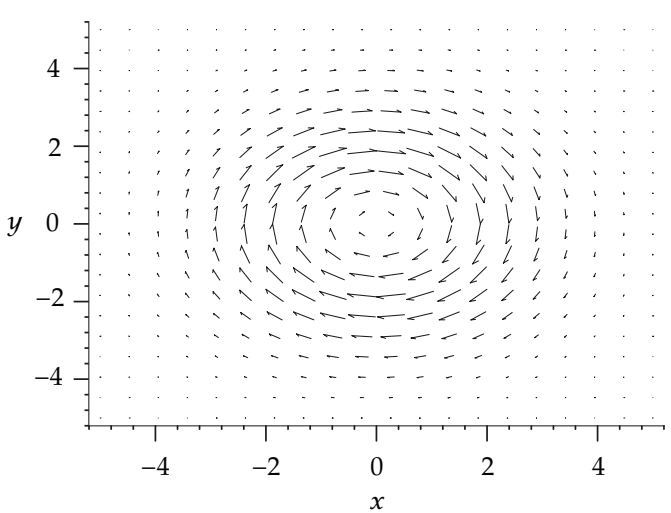

(b)

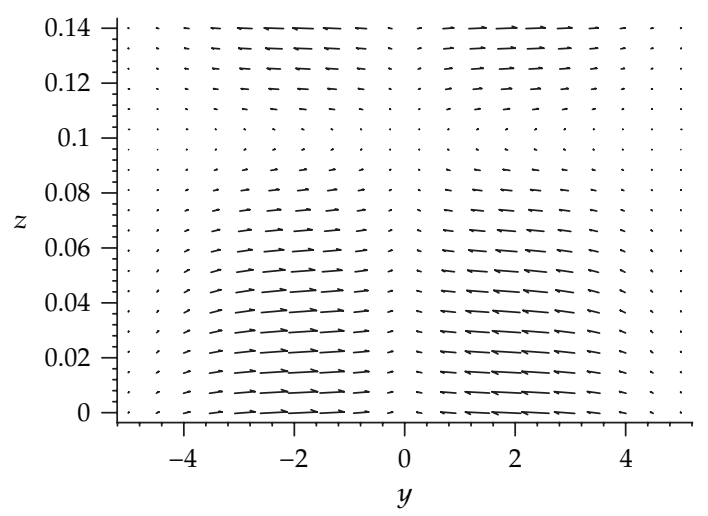

(c)

Figure 1: The profiles of the velocity field given by the horizontal velocities $u, v$ and the vertical velocity $w$ expressed by (6.5), (6.6), and (6.7), respectively, with the parameters (6.8) in the horizontal $x-y$ plane (a) at the bottom $z=0,(\mathrm{~b})$ at the top $z=0.14$, and (c) in the vertical $y-z$ plane with $x=0$.

\section{Summary and Discussions}

The symmetries of the rotational fluid model (1.1)-(1.5) are studied in three ways. Firstly, we directly study the Lie symmetries of the full system (1.1)-(1.5) for the fields $\{u, v, p, \rho, w\}$. The result shows us that the Lie symmetries do not contain arbitrary functions of $z$ and the time scaling is companied by a time-dependent rotation. Secondly, the Lie symmetries of the subsystem (1.1)-(1.3) for the fields $\{u, v, p\}$ exhibit eight arbitrary functions with five of them being arbitrary functions of $\mathrm{z}$ and others being arbitrary functions of $\{z, t\}$ while the time scaling invariance is still companied by a time-dependent rotation. Though these symmetries are the Lie symmetries for the subsystem $\{u, v, p\}$, they are generalized symmetries for the full system $\{u, v, p, \rho, w\}$. Finally, we enlarged the original system to an enlarged one which contains the stream function, four-dimensional vector forms $\vec{v}$ and $\vec{f}$ shown by (4.2) and the $4 \times 4$ symmetric tensors $\psi_{i j}$ and $p_{i j}$ shown by (4.3). For the enlarged system, the Lie symmetries display much abundant structure which possesses six arbitrary functions of $z$ and four arbitrary functions of $\{z, t\}$. It is shown that because of the entrance of the stream function, the time scaling invariance and time-dependent rotation invariance become independent potential symmetries which are nonlocal symmetries for the original system $\{u, v, p, \rho, w\}$ though they are local Lie symmetries for the enlarged system. 


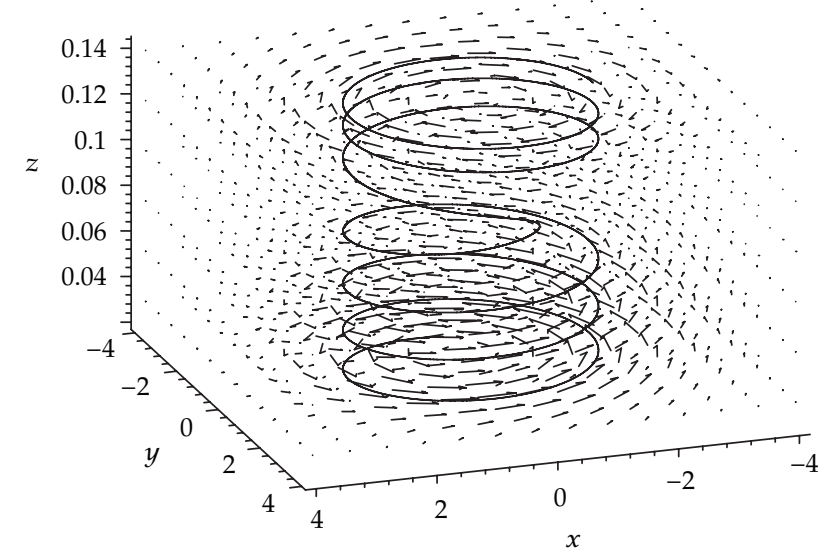

Figure 2: The-three dimensional spiral pattern of a typhoon vortex structure with the velocities $u, v$, and $w$ expressed by (6.5)-(6.8), respectively. The spiral line is a numerical solution of (6.9) with (6.5)-(6.8) and the initial conditions $x(0)=z(0)=0$ and $y(0)=2$.

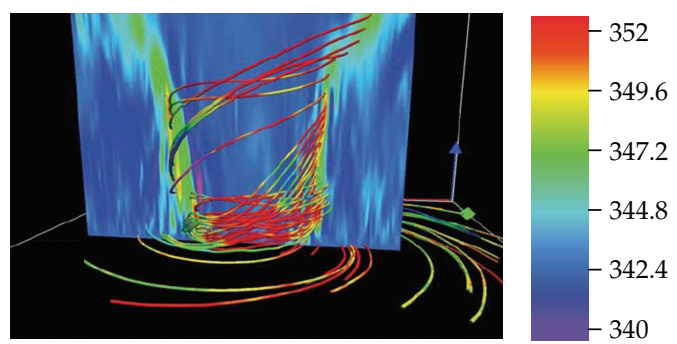

Figure 3: Picture of a recent high-resolution hurricane simulation using the WRF-ARW, cited from [15].

The corresponding finite symmetry transformation group related to both the generalized local symmetries and nonlocal symmetries for the subsystem $\{u, v, p\}$ are obtained because the symmetries are only Lie symmetries for the enlarged system. The finite symmetry transformation group can be used to find quite general solutions from simple ones. Especially a known trivial solution is transformed to produce a quite general vortex solution. A special form of the obtained vortex solution is used to qualitatively display the three-dimensional structure of cyclones.

All results of the paper in fact are based on the fact that system (1.1)-(1.5) is degenerate in certain sense. Namely, subsystem (1.1)-(1.3) involves the independent variable $z$ as a parameter. It is obvious that any local (i.e., generalized [1]) symmetry of subsystem (1.1)(1.3) can be extended to a local symmetry of the entire system (1.1)-(1.5) using (1.4) and (1.5) and the standard prolongation procedure. The same assertion is true for finite symmetry transformations and potential symmetry associated with the stream function as a potential. Similarly, any solution of subsystem (1.1)-(1.3) can be extended to a solution of the entire system (1.1)-(1.5) using (1.4) and (1.5) for defining $\rho$ and $w$. Moreover, the equation for the stream function coincides with the classical vorticity equation for an ideal incompressible fluid. Subsystem (1.1)-(1.3) is reduced to the two-dimensional Euler equations via simple transformation of the pressure involving the stream function. This is why the simplest algorithm for finding exact solutions system (1.1)-(1.5) is the following. Take any solution of the vorticity equation assuming that all constant and functional parameters additionally 
depend on the variable $z$. Then find the corresponding values of $u, v, p, \rho$, and $w$ using (4.1) and (1.2)-(1.5) respectively.

\section{Acknowledgments}

The authors are grateful for the referee's agreement to add the comment paragraph (the last paragraph of the paper) to the paper. This work was supported by the National Natural Science Foundation of China (nos. 10735030, 11175092, 10905038, and 40305009), the National Basic Research Program of China (nos. 2007CB814800 and 2005CB422301), the Specialized Research Fund for the Doctoral Program of Higher Education (no. 20070248120), SRF for ROCS, SEM and K. C. Wong Magna Fund in the Ningbo University.

\section{References}

[1] P. J. Olver, Applications of Lie groups to differential equations, vol. 107 of Graduate Texts in Mathematics, Springer, New York, NY, USA, 2nd edition, 1993.

[2] B. Fuchssteiner, "Application of hereditary symmetries tononlinear evolution equations," Nonlinear Analysis: Theory, Methods \& Applications, vol. 3, no. 6, pp. 849-862, 1979.

[3] B. Fuchssteiner, "The Lie algebra structure of nonlinear evolution equations admitting infinitedimensional abelian symmetry groups," Progress of Theoretical Physics, vol. 65, no. 3, pp. 861-876, 1981.

[4] W. X. Ma, "k symmetries and $\tau$ symmetries of evolution equations and their Lie algebras," Journal of Physics A, vol. 23, no. 13, pp. 2707-2716, 1990.

[5] C. Tian and Y. J. Zhang, "Bäcklund transformations for the isospectral and nonisospectral MKdV hierarchies," Journal of Physics A, vol. 23, no. 13, pp. 2867-2877, 1990.

[6] W. X. Ma, R. K. Bullough, P. J. Caudrey, and W. I. Fushchych, "Time-dependent symmetries of variable-coefficient evolution equations and graded Lie algebras," Journal of Physics A, vol. 30, no. 14, pp. 5141-5149, 1997.

[7] G. Bluman, A. F. Cheviakov, and N. M. Ivanova, "Framework for nonlocally related partial differential equation systems and nonlocal symmetries: extension, simplification, and examples," Journal of Mathematical Physics, vol. 47, no. 11, Article ID 113505, 23 pages, 2006.

[8] N. M. Ivanova, R. O. Popovych, C. Sophocleous, and O. O. Vaneeva, "Conservation laws and hierarchies of potential symmetries for certain diffusion equations," Physica A, vol. 388, no. 4, pp. 343-356, 2009.

[9] N. M. Ivanova and C. Sophocleous, "Conservation laws and potential symmetries of systems of diffusion equations," Journal of Physics A, vol. 41, no. 23, Article ID 235201, 14 pages, 2008.

[10] D. K. Lilly, "Stratified turbulence and the mesoscale variability of the atmosphere," Journal of the Atmospheric Sciences, vol. 40, no. 3, pp. 749-761, 1983.

[11] C. Sun, "A baroclinic laminar state for rotating stratified flows," Journal of the Atmospheric Sciences, vol. 65, no. 8, pp. 2740-2747, 2008.

[12] A. Bihlo and R. O. Popovych, "Lie symmetries and exact solutions of the barotropic vorticity equation," Journal of Mathematical Physics, vol. 50, no. 12, Article ID 123102, 12 pages, 2009.

[13] R. Berker, "Intégration des équations du mouvement d'un fluide visqueux incompressible," in Handbuch der Physik, Bd. VIII/2, pp. 1-384, Springer, Berlin, Germany, 1963.

[14] V. K. Andreev and A. A. Rodionov, "Group classification and exact solutions of equations of plane and rotational-symmetric flow of an ideal fluid in Lagrangian coordinates," Journal of Differential Equations, vol. 24, no. 9, pp. 1577-1586, 1988.

[15] A. Norton, Y. S. Chen, and J. Clyne, Analysis and Visualization of High-Resolution WRF Hurricane Simulation Using VAPOR, National Center for Atmospheric Research, Boulder, Colo, USA.

[16] S. Y. Lou, M. Jia, X. Y. Tang, and F. Huang, "Vortices, circumfluence, symmetry groups, and Darboux transformations of the $(2+1)$-dimensional Euler equation," Physical Review E, vol. 75, no. 5, Article ID 056318, 2007. 


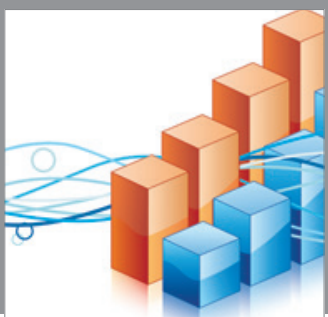

Advances in

Operations Research

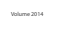

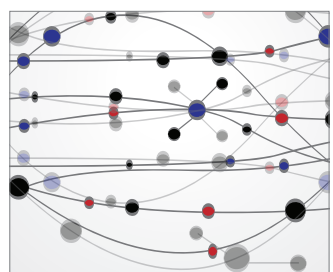

\section{The Scientific} World Journal
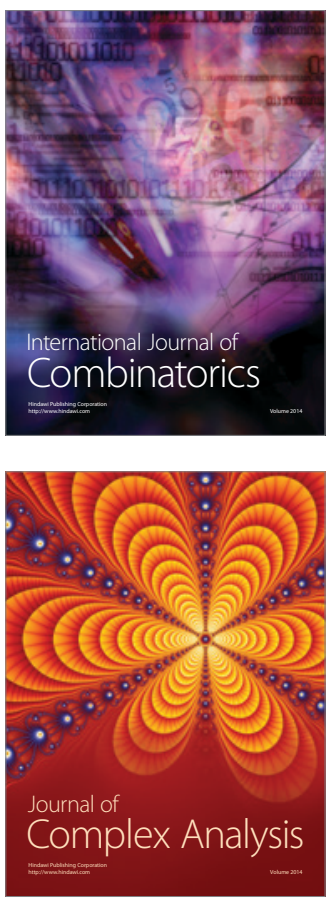

International Journal of

Mathematics and

Mathematical

Sciences
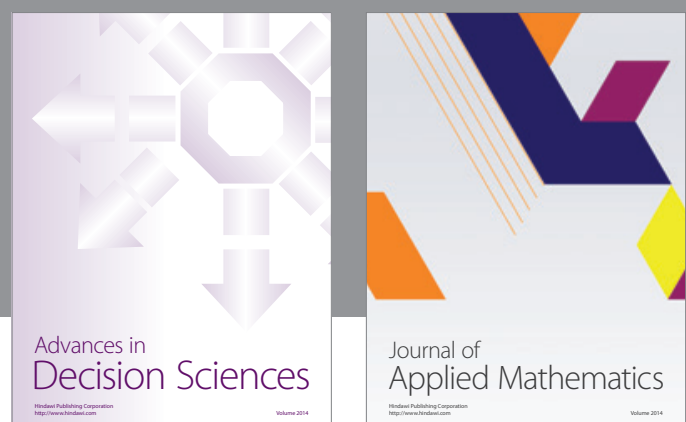

Journal of

Applied Mathematics
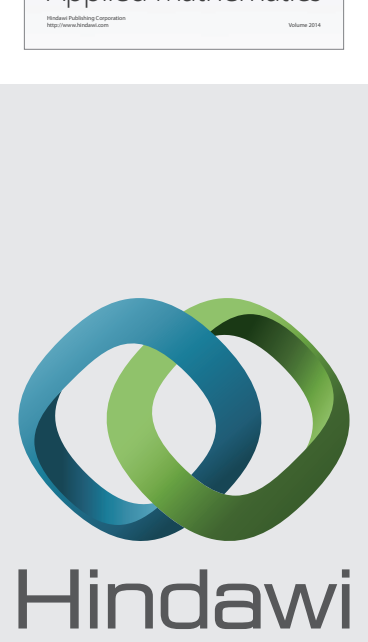

Submit your manuscripts at http://www.hindawi.com
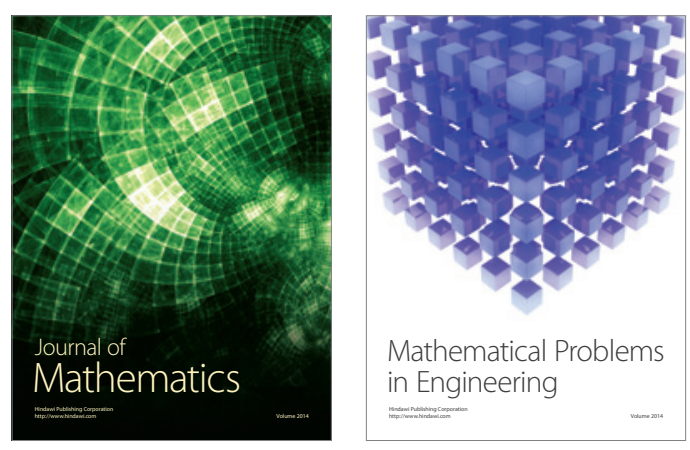

Mathematical Problems in Engineering
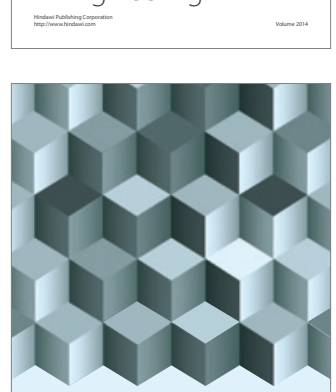

Journal of

Function Spaces
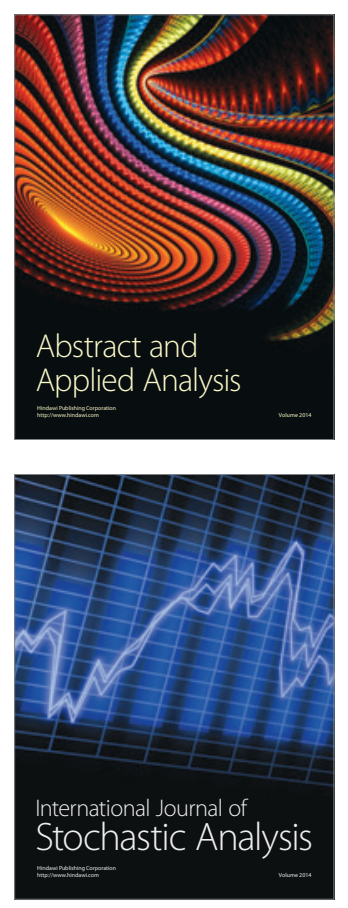

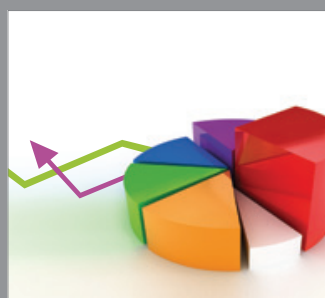

ournal of

Probability and Statistics

Promensencen
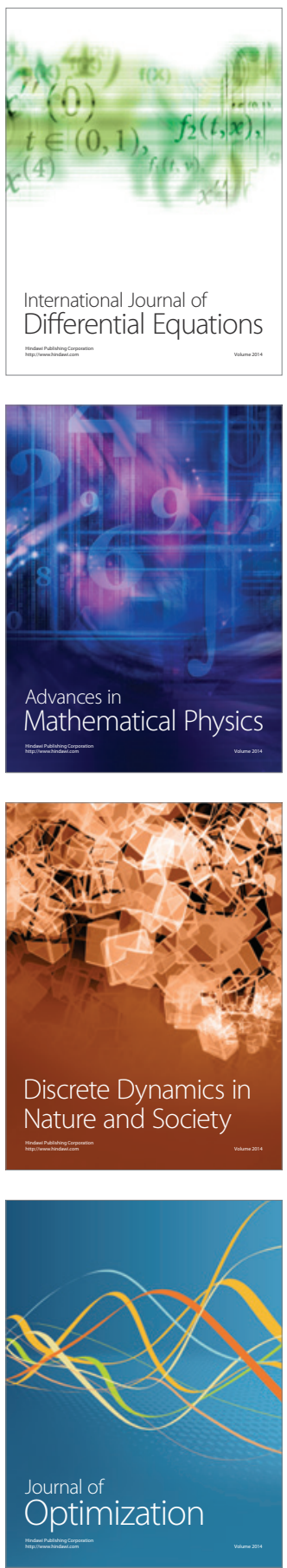